\title{
Wahr oder falsch? Eine empirische Untersuchung zur Wahrnehmung von »Fake News « und echten Nachrichten in der politischen Kommunikation
}

\author{
Ralf Hohlfeld
}

\section{Abstract}

Auf der Basis zweier Onlinebefragungen mit quasi-experimentellem Untersuchungsansatz in den Jahren 2017 und 2020 wurde analysiert, welche Bedeutung »Fake News « in der wahlberechtigten Bevölkerung in Deutschland zugeschrieben wird (Problemsensibilität), inwieweit die Fähigkeit bei Internetnutzern ausgebildet ist, Falschmeldungen von echten Nachrichten zu unterscheiden (Problemlösungskompetenz) und welche Faktoren das Erkennen von »Fake News" begünstigen (Problembewältigung). Bei großer Problemsensibilität unter Wirksamkeit des Third-Person-Effekts und mäßiger Problemlösungskompetenz, »Fake News"identifizieren zu können, schält sich als zentraler Befund der Illusory Truth Effect heraus. Dieser ist dafür verantwortlich, dass Menschen, die mit einer Meldung schon einmal konfrontiert waren, dieser mit einer höheren Wahrscheinlichkeit Glauben schenken, auch wenn diese offenkundig unplausibel und falsch ist.

\section{Einführung}

Desinformation ist eine Konstante der politischen Kommunikation. Die geschichtlichen Beispiele für bewusst lancierte Falschmeldungen sind Legion (Keil \& Kellerhoff, 2006; siehe auch den Beitrag von Walter Hömberg), ihre konkreten Wirkungen sind jedoch unklar. Im Zuge des Wahlkampfs zur US-Präsidentschaftswahl 2016 hatte die beabsichtigte Verbreitung von Nachrichten zur Täuschung der Bevölkerung eine neue Qualität erreicht. Im Konzert mit sogenannten durch Hackerangriffe geleakten Informationen und der Verbreitung durch Social Bots in sozialen Netzwerken haben »Fake News« Einfluss auf den seinerzeit überraschenden Wahlausgang zugunsten Donald Trumps genommen. Seitdem grassiert die Befürchtung, die politische Kommunikation könnte speziell in Wahlkampfzeiten immer wieder 
Opfer von orchestrierten und gelenkten Maßnahmen der Desinformation werden.

Sicher ist, dass willkürliche Falschinformationen seit Jahren in großer Zahl in Umlauf gebracht werden (Beermann, 2017). Lancierte Falschmeldungen spielen insbesondere in der politischen Kommunikation eine wichtige Rolle, da sie das öffentliche Meinungsbild und somit auch die politischen Entscheidungsprozesse beeinflussen können. »Fake News« als aktuelle Erscheinungsform von Desinformation (Zimmermann \& Kohring, 2018) sind zu neuen Paradigmen politischer Kommunikation geworden.

Falsch- und Desinformationen und deren gezielte Verbreitung haben das Potential, politische Wahlausgänge sowie die öffentliche Meinung zu beeinflussen und zu manipulieren. Auf politischer, wirtschaftlicher und gesellschaftlicher Ebene wird deutlich, dass der Informationskrieg (engl. Information Warfare) einem elektronischen Konflikt entspricht, in dem Informationen einen strategischen Gewinn darstellen (Schwartau, 1996): Auf Basis falscher Informationen wird die Wahrnehmung der Rezipienten bei Nichterkennen manipuliert, was Auswirkungen auf die Meinungsbildung und die darauffolgenden Handlungen der Rezipienten haben kann.

Desinformationskampagnen gehen oft Hand in Hand mit politischer Propagandakommunikation und verfolgen das Ziel, »bei den Empfängern eine bestimmte Wahrnehmung von Ereignissen oder Meinungen zu [sic] auszulösen, nach der neue Informationen und Sachverhalte in den Kontext einer ideologiegeladenen Weltsicht eingebettet werden « (Bussemer, 2008, 13). Bei der Verbreitung propagandistischer und falscher Inhalte spielen vor allem soziale Netzwerke eine große Rolle. Denn aufgrund der Funktionslogik sozialer Netzwerke ist es möglich, gezielt propagandistische »Fake News« an dafür empfängliche Personen- und Interessensgruppen zu streuen. Durch das Teilen erscheint die Information schließlich auch im Social-Media-Feed ihrer Kontakte und verteilt sich durch das Peer-to-Peer-Netzwerk immer weiter (Wardle, 2017).

\section{Funktionen und Definition von Fake News}

In der Forschung ist der Begriff »Fake News« nicht unumstritten, weder was seine begriffliche Präzision angeht noch hinsichtlich seiner politischen Instrumentalisierung. Deshalb wird unterdessen der Begriff der aktuellen Desinformation favorisiert (Zimmermann \& Kohring, 2018).

Dessen ungeachtet herrscht eine gewisse Uneinigkeit darüber, welche publizistischen Formen konkret unter die Begriffe »Fake News« oder aktu- 
elle Desinformation fallen, wenn es um die politischen Auswirkungen geht. Tandoc Jr., Lim und Ling (2018) unterscheiden etwa die sechs Kategorien Nachrichtensatire, Nachrichtenparodien, Fabricated News, Bildmanipulation, Werbung und PR sowie Propaganda. Wardle (2017) nennt dagegen sieben Formen von Fehl- und Desinformation: Erfundene Inhalte, betrügerische Inhalte, irreführende Inhalte, überarbeitete Inhalte, falsche Zusammenhänge, falsche Verknüpfungen und Satire/Parodie, die sie in einem zweidimensionalen Kontinuum anordnet. Der eine Pol markiert dabei die zielgerichtete Falschinformation mit der Absicht zu täuschen: Betrügerische Inhalte mit falschen Quellen, die lediglich vorgeben authentisch zu sein, sowie überarbeitete Inhalte, die zum Zweck der Täuschung erstellt wurden, sind dabei im Bereich der zielgerichteten »Fake News « zu verorten (Wardle, 2017). Dabei besonders zu berücksichtigen sind die erfundenen Inhalte, also Nachrichten mit erfundenen beziehungsweise überwiegend falschen Inhalten, die »mit der Absicht erstellt wurden, zu täuschen oder Schaden zu verursachen« (Wardle, 2017). Jene intendierten »Fake News«, die ausschließlich mit dem Ziel der Täuschung und Manipulation der Rezipienten erstellt werden, sind für den vorliegenden Forschungskontext relevant. Irrelevant sind dagegen die Formate mit geringer Täuschungsabsicht wie Satire oder Parodie - Nachrichten, die mit Stilmitteln wie Humor, Ironie oder Übertreibungen versehen sind, jedoch grundsätzlich nicht produziert wurden, um zu schaden, sondern nur um mediale Aufmerksamkeit zu erregen. Diese markieren den anderen Pol.

Inhalte mit irreführenden Informationen, falschen Verknüpfungen sowie falschen Zusammenhängen bewegen sich Wardle (2017) zufolge im Bereich der geringen bis mittleren Täuschungsabsicht, jedoch sind diese oft besonders wirksam. Desinformation kann nämlich auch als eine Mischung von wahren und frei erfundenen Tatsachen verstanden werden (Van Herpen, 2016).

Zwar können auch irreführende Nachrichten und Schlagzeilen, die auf Clickbaiting abzielen sowie tendenziöse und einseitige Meldungen zur Täuschung der Rezipienten beitragen (Chen, Rubin \& Conroy, 2015). In dieser Studie wird jedoch Gelfert (2018) gefolgt, der dafür plädiert, vor allem »echte Fake News« von Falschmeldungen, Satire und ähnlichen Formaten zu unterscheiden: »Fake news is the deliberate presentation of (typically) false or misleading claims as news, where the claims are misleading by design« (Gelfert, 2018, 108). 


\section{Forschungsstand}

Zu den Faktoren, die Menschen anfällig für Desinformation machen, gehören unter anderem Persönlichkeitsvariablen. Bei der Suche nach einem Personentypus, der offenbar besonders anfällig dafür ist, Falschmeldungen und Verschwörungsmythen für wahr zu halten, haben Graeupner und Coman (2017) gesellschaftliche Außenseiter beziehungsweise soziale Exklusion identifiziert. In einem Laborexperiment konnte nachgewiesen werden, dass Teilnehmer, die sich ausgeschlossen fühlten, stärker nach dem Sinn des Lebens suchten und sich dabei anfälliger für Verschwörungsmythen und Aberglauben zeigten.

In eine ähnliche Richtung gehen die Forschungen von Allcott und Gentzkow (2017), die sich mit den soziodemographischen Variablen im Verhältnis zur Mediennutzung und dem Vertrauen in die dort publizierten Informationen in den sozialen Medien beschäftigt haben. Die Autoren fanden in ihrer Untersuchung drei Distinktionskriterien, die eine Erklärung für die Fähigkeit, »Fake News« zu erkennen, liefern. 1) Menschen, die mehr Zeit mit dem Konsum von Medien verbringen, 2) Mediennutzer mit höherer Bildung und 3) ältere Menschen können Meldungen besser auf ihren Wahrheitsgehalt hin einschätzen. Ferner konnten Studienteilnehmer, die soziale Medien als ihre Hauptquelle für Nachrichten über politische Wahlen angeben, den Wahrheitsgehalt der Nachrichten besser einschätzen und Falschmeldungen eher identifizieren. Diese Befunde stehen allerdings im Widerspruch zu anderen Erhebungen, die eine Bevorzugung klassischer Nachrichtenmedien in Abgrenzung zu alternativen Nachrichtenseiten (Tsfati \& Ariely, 2014; Tsfati \& Cappella, 2003) als Prädikator ansehen, der die Nutzer befähigt, die Glaubwürdigkeit von Nachrichtenquellen im Internet korrekt zu beurteilen.

Auch für konsonanztheoretische Erklärungen und den Einfluss selektiver Medienzuwendung konnten Allcott und Gentzkow (2017) Anhaltspunkte finden: Anhänger sowohl der Demokraten als auch der Republikaner glaubten Artikeln eher, die ihrer Weltanschauung entsprechen, als solchen, die dies nicht tun, was auch von einer Untersuchung von Metzger, Hartsell und Flanagin (2015) bestätigt wurde, die herausfanden, dass Rezipienten diejenigen Quellen als glaubwürdiger ansehen, die mit ihrer Einstellung übereinstimmen - allerdings ist hier zu berücksichtigen, dass Liberale und Anhänger der Demokratischen Partei ein grundsätzlich höheres Vertrauen in die Medienberichterstattung haben (Lee, 2010).

Pennycook, Cannon und Rand (2017) von der Yale University fanden Hinweise, dass unter anderem kognitive Mechanismen für die Frage, ob 
man Meldungen Glauben schenkt, verantwortlich sind, zum Beispiel die Vertrautheit. Sie beziehen sich in ihrer Studie auf eine frühere Forschung zum illusorischen Wahrheitseffekt, die aufzeigt, dass Vertrautheit den wahrgenommenen Wahrheitsgehalt von plausiblen und harmlosen (aber nicht notwendigerweise wahren) Aussagen erhöht. Daran schließt sich die Frage an, ob sich dieser Effekt ebenfalls auf offenkundig unplausible Aussagen erstreckt. Dazu wurden Probanden im Rahmen eines Experiments mit gefälschten und echten Nachrichten konfrontiert. Diese erachteten offenkundig unplausible Meldungen für wahr, wenn sie von diesen schon gehört oder gelesen hatten. Darüber hinaus wurde auch eine erhöhte Glaubwürdigkeit für bereits bekannte, aber gefälschte Schlagzeilen evident, selbst wenn die Nachrichten durch sogenannte Fact Checks als »Fake News« eingestuft wurden oder mit der politischen Ideologie des Rezipienten nicht vereinbar sind. Der Effekt trat sogar dann auf, wenn sich die Probanden nicht mehr bewusst erinnerten, die falsche Schlagzeile schon einmal gelesen zu haben (Pennycook et al., 2017).

Daraus folgt, dass Vertrautheit heuristisch verwendet wird, um auf den Wahrheitsgehalt zu schließen. Die Verbreitung von gefälschten Nachrichten wird durch anhaltende, niedrige kognitive Prozesse gestützt, die auch weniger plausible und sehr parteiische Forderungen mit Hilfe von Wiederholungen glaubwürdiger machen. Die Ergebnisse deuten darauf hin, dass informationsbasierte Filterblasen und politische Echokammern nicht nur Menschen von anderen Meinungen isolieren, sondern auch Brutstätten für offensichtlich falsche, reißerische und politisierte Nachrichten schaffen. So entsteht ein Teufelskreis, in dem das Teilen von »Fake News« die Glaubwürdigkeit erhöhe, was wiederum zu vermehrtem Teilen der Nachrichten führt und so die Glaubwürdigkeit abermals erhöhe (Pennycook et al., 2017).

An diesem Teufelskreis sind in den USA vor allem ältere Social-MediaNutzer beteiligt (Guess, Nagler \& Tucker, 2019): Während auf der Basis der Untersuchung von 1331 Facebook-Profilen festgestellt werden konnte, dass unter den 18- bis 29-jährigen Amerikanern nur drei Prozent »Fake News « weiterleiten, sind es bei den über 65-Jährigen elf Prozent. Eine ähnliche Diskrepanz konnte auch bei den politischen Einstellungen erkannt werden. Hier sind es politisch konservative Nutzer - vor allem Trump-Anhänger - die Falschmeldungen mit ihren Freunden teilen. 


\section{Forschungsfragen und Hypothesen}

Aus dem zusammengefassten Forschungsstand geht hervor, dass Desinformationen Bürgerinnen und Bürger eines demokratischen Staates irritieren, weil diese keine Sicherheit in der Beurteilung der Glaubwürdigkeit der Meldungen und ihrer Quellen besitzen. Insofern können »Fake News« politische Entscheidungsprozesse verfälschen und zur Destabilisierung von pluralistischen Gesellschaften beitragen. Um das Ausmaß der individuellen und sozialen Wirkungen abschätzen zu können, muss man zunächst die Fähigkeit der Nutzer analysieren, Informationen (als Nachrichten) von Desinformation (als »Fake News«) unterscheiden zu können. Deshalb geht es in diesem Kontext um die Wahrnehmung von Meldungen in zwei Aggregatsstufen: als Nachrichten und als Desinformation.

Die vorliegende Studie beschäftigt sich daher mit dem...

Bewusstsein der deutschen Bevölkerung bezüglich »Fake News«, der Fähigkeit, diese identifizieren zu können und der Frage, welche Bedeutung den Falschmeldungen zugeschrieben wurde und wird.

Im Einzelnen ergeben sich aus dem Forschungsstand folgende Fragen:

1. Inwieweit sind Internetnutzer in der Lage, »Fake News« zu erkennen?

2. Hat die Bekanntheit einer Meldung Einfluss auf die Identifizierbarkeit als wahr oder falsch?

3. Welche Eigenschaften und Verhaltensweisen beeinflussen die Eignung der Nutzer, »Fake News « von Nachrichten unterscheiden zu können?

4. Wie gehen Nutzer mit identifizierten Falschmeldungen um?

5. Versehen sie sie mit Aufmerksamkeit, indem sie diese teilen oder kommentieren?

6. Fühlen die Nutzer sich von der Desinformation beeinflusst - insbesondere in Zeiten politischer Wahlen?

7. Ist bei der gefühlten Beeinflussung eine Zweit- und/oder Dritt-Personen-Wahrnehmung (Second-/Third-Person-Effekt) wirksam?

Einige dieser Forschungsfragen lassen sich durch deduktiv aus dem Forschungsstand identifizierte Anhaltspunkte in Form von Annahmen operationalisieren, die in dieser Studie getestet werden. Dabei handelt es sich um folgende Hypothesen: 


\section{Haupthypothese:}

H1: Bekannten Meldungen wird ein höherer Wahrheitsgehalt zugeschrieben als unbekannten Meldungen, auch wenn die schon bekannte Meldung eine Falschmeldung ist (Pennycook et al., 2017).

\section{Hypothesenkomplex A: Einfluss soziodemographischer Eigenschaften}

H2: Es besteht ein positiver Zusammenhang zwischen höherer Bildung und dem Erkennen von »Fake News" (Allcott \& Gentzkow, 2017).

H3: Je höher das Alter der Befragten ist, desto eher sind sie in der Lage, »Fake News« zu identifizieren (Allcott \& Gentzkow, 2017).

\section{Hypothesenkomplex B: Einfluss verhaltensbezogener Eigenschaften}

H4.1: Je höher der Medienkonsum der Befragten, desto eher sind sie in der Lage, "Fake News" zu identifizieren (Allcott \& Gentzkow, 2017; Pennycook et al., 2017).

H4.2: Die Art der genutzten Online-Medien zur politischen Information hat einen Einfluss auf die Fähigkeit, »Fake News" zu identifizieren (Tsfati \& Ariely, 2014; Tsfati \& Cappella, 2003; Allcott \& Gentzkow, 2017).

H5.1: Es besteht ein positiver Zusammenhang zwischen politischem Engagement und dem Erkennen von »Fake News" (Smith, 2013).

H5.2: Die politische Gesinnung beziehungsweise Orientierung hat Einfluss auf das Erkennen von »Fake News" (Lee, 2010; Metzger et al., 2015; Allcott \& Gentzkow, 2017).

\section{Hypothesenkomplex C: Zweit- und Drittpersonen-Wahrnehmung bei der Beeinflussbarkeit}

H6.1: Die Befragten sind der Ansicht, dass sie selbst weniger von »Fake News" beeinflusst werden als die restliche Bevölkerung (Dohle, 2016; Rössler, 2009; YouGov, 2017).

H6.2: Die Befragten sind der Ansicht, dass ihr direktes soziales Umfeld weniger von »Fake News" beeinflusst wird als die restliche Bevölkerung (Dohle, 2013). 


\section{Methode}

Als Untersuchungsmethode wurde ein experimentelles Studiendesign gewählt, das in eine quantitative Online-Befragung eingebettet wurde. Es wurden echte und erfundene Meldungen (darunter auch etablierte »Fake News«) in den Fragebogen integriert, um möglichst realitätsnah die Einschätzung des Wahrheitsgehalts von Meldungen auf sozialen Plattformen wie Facebook oder Twitter zu messen. Den Probanden wurden jeweils zu Beginn des Fragebogens aus einem Pool von insgesamt 20 Meldungen fünf beziehungsweise sechs wahre Nachrichten und fünf beziehungsweise sechs »Fake News" in randomisierter Reihenfolge vorgelegt, die diese als wahr oder falsch identifizieren mussten. Außerdem sollten die Befragten angeben, ob sie auf diese Nachricht in sozialen Netzwerken zustimmend oder ablehnend reagieren würden und ob sie von dieser Nachricht bereits einmal gehört haben.

Die Inhalte der gewählten Meldungen - sowohl die der erfundenen als auch die der wahren - behandeln relevante Themen, die zum Zeitpunkt der Studie aktuell in den Nachrichten behandelt wurden. Vorrangig beziehen sich umgeschriebene Meldungen auf den Klimawandel, die Migrations- und Geflüchtetenthematik sowie auf den Rechtsextremismus. Die Meldungen wurden mit einer Schlagzeile und einem dazugehörigen Foto angezeigt. Abgedeckt wurde das Spektrum von irreführenden Inhalten über überarbeitete Inhalte bis zu erfundenen Inhalten und von falschen Verknüpfungen bis zu falschen Zusammenhängen.

Die Studie fand zu zwei Zeitpunkten statt. Sie ging erstmals in der zweiten Julihälfte 2017 - also rund acht Wochen vor der Bundestagswahl - für zwei Wochen ins Feld ${ }^{1}$ und wurde zweieinhalb Jahre später im Januar 2020 mit im wesentlichen identischem Messinstrument wiederholt ${ }^{2}$. Mit dem zweiten Messzeitpunkt sollte überprüft werden, ob sich das Antwortverhalten in einer Phase ohne politische Wahlkämpfe von der heißen Phase des

1 An der Studie 2017 waren folgende Personen beteiligt: Bastian Alpers, Pia-Rebekka Arend, Angelica Balbuena, Astrid Biedermann, Chrestien Delonge, Katharina Elisabeth Eckert, Lorenz Feldrapp, Vanessa Fernbacher, Anja Fichtl, Susanne Frank, Patricia Germann, Franziska Hofmann, Alex Klaus, Jennifer Lax, Daniel Lorenzer, Angelique Markus, Moritz Matzewitzki, Mirjam Mittermeyer, Lukas Peh, Julian Reitmeier, Maria Richtsfeld, Ramona Rumberger, Kilian Schmidt, Christopher Schmitt, Steffen Schnappauf, Tim Schöfer, Madeline Schürz, Rosario Sicali, Viktoria Tanchynets, Jule Trepmann, Verena Weber.

2 Mitglieder der Forschungsgruppe 2020 waren: Ilenia Braglia, Aqib Butt, Eva Haberzettel, Anja Krieger, Adriana Malkanova, Pia Ohnheiser, Friederike Peschek, Bianca Scharnberger, Hannah Schmid, Franziska Vogl, Franzisca Weny, Paulin Zeichinger. 
Wahlkampfs unterscheidet. Die Studien wurden im Rahmen von Lehrforschungsprojekten im Masterstudiengang Medien und Kommunikation an der Universität Passau durchgeführt; die Forscherteams haben sich dadurch naturgemäß unterschieden, Kontinuität konnte über den Studienleiter sichergestellt werden. Beiden Feldphasen ging jeweils eine intensive Pretestphase voraus, in der die Items der Befragung getestet wurden. Die Fragebögen, die mit SoSci Survey programmiert wurden, sind im Anschluss an einen Pretest (mit jeweils $\mathrm{n}=25$ ) mittels Convenience Samples distribuiert worden, wobei neben den sozialen Netzwerken der Forschergruppe eine Vielzahl von Multiplikatoren wie Medienhäuser und Landeszentralen für politische Bildung genutzt wurden; für die zweite Welle konnte zusätzlich noch auf Onlineportale wie Survey Circle und Poll Pool zurückgegriffen werden. Für das 2017er-Sample konnten 1.021 vollständig ausgefüllte Fragebögen realisiert werden, 2020 waren es insgesamt 1.042 .

\section{Befunde}

Beide Befragtengruppen sind stark in Richtung einer jüngeren (Altersdurchschnitt 2017: 32,1 Jahre; 2020: 27,4 Jahre), weiblichen (2017: 56,6 Prozent; 2020: 63,9 Prozent), formal höher gebildeten (Anteil Studierende 2017: 39,3 Prozent; 2020: 66,5 Prozent) und politisch progressiveren Population verzerrt, die regional überwiegend aus Bayern (2017: 68,2 Prozent; 2020: 63,8 Prozent) und dem restlichen Süddeutschland stammt. Alle Befragten sind in Deutschland wahlberechtigte Bürgerinnen und Bürger, die über 18 Jahre alt sind.

Das politische Interesse und die politische Orientierung wurden in den beiden Studien unterschiedlich erhoben. Im Sample 2017 wurden die Teilnehmer gefragt, ob sie an der Bundestagswahl teilnehmen werden. 90,3 Prozent bejahten, 7,7 Prozent waren noch unschlüssig und zwei Prozent gaben an, nicht wählen zu gehen. 48 Prozent hatten im Juli 2017 schon ihre Entscheidung getroffen, wen sie im September wählen wollen, 52 Prozent waren noch unschlüssig. Die Sonntagsfrage zeigte eine weitgehende Übereinstimmung mit dem späteren Ausgang der Bundestagswahl 2017, wobei die Grünen leicht überrepräsentiert und die AfD stark unterrepräsentiert war. 30 Prozent der Befragten gaben an, politisch engagiert zu sein. Im Sample 2020 wurde das politische Interesse skaliert abgefragt, das arithmetische Mittel $\bar{x}$ liegt bei 4,26 ( $1=$ sehr niedrig, $6=$ sehr hoch $)$, tendenziell schätzen die Befragten ihr eigenes politisches Interesse (bei einem Skalenmittelpunkt von 3,5) eher hoch ein, 15 Prozent der Befragten gaben hier an, ein sehr 
hohes Interesse an Politik zu besitzen, sechs Prozent waren Mitglied einer politischen Partei. Rund drei Viertel der Befragten gaben an, zumindest hin und wieder Programme von Parteien im Vorfeld von Wahlen zu lesen. Die politische Einstellung weist bei einem Skalenmittelpunkt von 3,5 mit $\bar{x}=$ 2,77 eine Tendenz nach links aus ( $1=$ links; $6=$ rechts $)$.

Nach politischer Information im Internet wurde im vorliegenden Befragtensample der Studie von 2020 in erster Linie in den Onlineangeboten von Printmedien (78 Prozent), den Onlineangeboten von Rundfunksendern (67 Prozent) und auf den Seiten von sozialen Netzwerken wie Facebook und Instagram (61 Prozent) gesucht. Eine geringere Rolle spielten Videoportale (32 Prozent), News-Aggregatoren (22 Prozent) und Twitter (17 Prozent), gefolgt von Instant-Messaging-Diensten wie WhatsApp und Snapchat (15 Prozent). In der Onlinebefragung 2017 war das Internet die meistgenutzte Plattform: 97 Prozent gaben an, mindestens mehrmals wöchentlich das Internet zu nutzen; 67 Prozent gaben diese Nutzung für das Radio an, für das Fernsehen waren es 64 Prozent, für die Zeitung nur 29 Prozent.

Die Befragten der 2017er Feldphase erkannten im Schnitt bei einem Mittelwert von 3,2 gerade einmal die Hälfte der sechs nach dem Zufallsprinzip präsentierten »Fake News« zweifelsfrei, was grundsätzlich auf eine gewisse Anfälligkeit für Desinformation schließen lässt. Nur sieben Prozent der Probanden waren in der Lage, alle sechs Falschmeldungen zu enttarnen. Korrekterweise als »Fake News« entlarvt wurden die randomisiert eingespielten tatsächlichen Falschmeldungen durchschnittlich von knapp 60 Prozent der Teilnehmer; jeder sechste bis siebte Proband jedoch saß der jeweiligen Falschmeldung auf, der Rest war sich unsicher.

Mit Blick auf die einzelnen »Fake News«-Stimuli schwankten die Werte beträchtlich: Die Meldung, dass Bundeskanzlerin Angela Merkel sich mit dem Brüssel-Attentäter auf einem Selfie hat ablichten lassen, glaubten nur fünf Prozent der Befragten. Dagegen hielten 35 Prozent der Befragten die vor allem im Umfeld der AfD vielfach geteilte Falschmeldung für glaubwürdig, wonach die Grünen-Politikerin Renate Künast zum Mord an einer Studentin in Freiburg gesagt habe: »Der traumatisierte junge Flüchtling hat zwar getötet, man muss ihm aber jetzt trotzdem helfen.« Nur bei dieser Falschmeldung glaubten mehr Teilnehmer der »Fake News«, als diese sie anzweifelten (25 Prozent). Das Ergebnis spricht dafür, dass die Wirkung von »Fake News«, die einen bestehenden Nachrichtenkern nutzen (Verständnis einer Politikerin für straffällige Geflüchtete) und diesen umdeuten und abwandeln (Hilfe für einen Mörder) im Vergleich zu gänzlich frei erfundenen Meldungen größer ist. 
Wie Abbildung 1 zeigt, ist die Anzahl der richtig erkannten »Fake News« in der Stichprobe normalverteilt. Für den Bereich des Erkennens von »Fake News« wurde anschließend ein Score mit dichotomer Ausprägung gebildet. Dieser wurde aus den Daten extrahiert, wie viele der vorgelegten Falschmeldungen erkannt und von den Befragungsteilnehmern richtig eingeordnet wurden. Die Teilnehmer wurden anhand der Ergebnisse in die zwei Gruppen »schlecht im Erkennen« (= null bis drei erkannte FN: 55,4 Prozent) und "gut im Erkennen« (vier bis sechs erkannte FN: 44,6 Prozent) unterteilt.

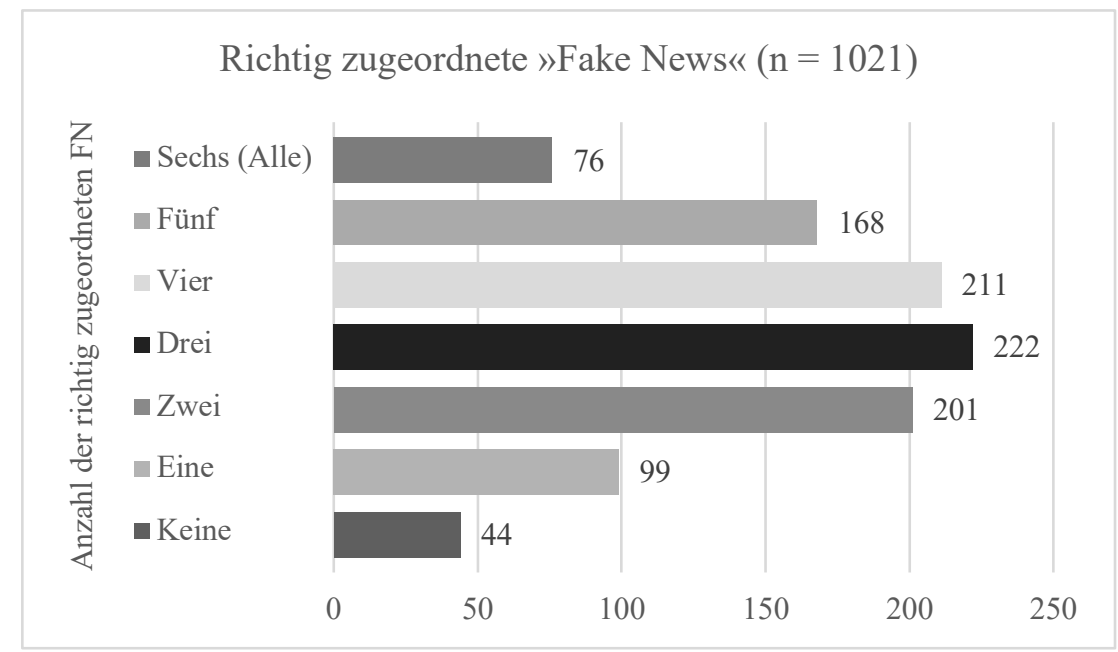

Abbildung 1: Anzahl der richtig zugeordneten Meldungen pro Person

Die Teilnehmenden wurden zudem befragt, ob sie bereit wären, die zur Beurteilung vorliegenden Meldungen in den sozialen Netzwerken zu teilen. Gegen eine prinzipiell hohe Viralität von »Fake News« spricht der vorliegende Befund, dass nur 1,5 Prozent der Befragten die Meldung bei Facebook und Twitter in zustimmender Absicht teilen oder liken würde. Umgekehrt wären aber im Durchschnitt der untersuchten Fälle zehn Prozent der Probanden bereit, erkannte »Fake News« mit kritischen Kommentaren zu versehen und weiterzuleiten. Dies war vor allem bei »Fake News« zu scheinbar straffälligen Geflüchteten der Fall.

In der Nachfolgeuntersuchung von 2020 wurde zudem gefragt, welche Methoden die Teilnehmer nutzen, um im Zweifelsfall den Wahrheitsgehalt einer Meldung zu überprüfen. Die große Mehrheit von 89 Prozent zieht mehrere Artikel zum Thema heran, 66 Prozent prüfen die Ausdrucksweise auf mögliche satirische Elemente, 44 Prozent überprüfen den Autor, 39 Prozent das Datum und 31 Prozent prüfen den inhaltlichen Aufbau des Artikels. 
Seltener wird die URL auf Plausibilität gecheckt (26 Prozent) oder die Möglichkeit der Bilderrückverfolgung bei Google (13 Prozent) genutzt. Immerhin zwölf Prozent beziehen Initiativen, die »Fake News« enttarnen, ein. Der Bekanntheitsgrad der Initiativen ist recht schmal: 18 Prozent der Befragten kennen Correctiv, 16 Prozent den BBC Reality Check, 12 Prozent FactCheckEU, fünf Prozent StopFake und vier Prozent Bellingcat. Drei Prozent der Teilnehmer gaben an, den Service Is it a Fake? zu kennen, der vom Forschungsteam erfunden war.

Zu den zentralen Aspekten der Studie (hier 2020) zählt die Frage, ob die Teilnehmer in ihrer Meinungsbildung von »Fake News « beeinflusst worden sind. Bei einer Skala von 1 (trifft überhaupt nicht zu) bis 5 (trifft voll und ganz zu) liegt der Mittelwert bei $\bar{x}=2,71$. Geringfügig kleiner ist mit $\bar{x}=$ 2,54 der Wert für die Aussage: Ich habe mir durch Nachrichten eine Meinung zu einem politischen Thema gebildet, aber hinterher stellte sich raus, dass die Informationen nicht korrekt waren.

Dagegen können sich sehr viele Teilnehmer durchaus vorstellen, schon einmal zumindest unbewusst von »Fake News« beeinflusst worden zu sein, das arithmetische Mittel liegt hier bei $\bar{x}=3,82$ (bei einem Skalenmittelpunkt von 3).

\section{Hypothesenprüfung}

Bei der Hypothesenprüfung folgt die Darstellung aus Gründen der Übersichtlichkeit den Datenanalysen der Ausgangsstudie von 2017. Falls für 2020 grob abweichende Befunde vorliegen, wird dies im Folgenden angezeigt und diskutiert.

H1: Bekannten Meldungen wird ein höherer Wahrheitsgehalt zugeschrieben als unbekannten Meldungen, auch wenn die schon bekannte Meldung eine Falschmeldung ist.

Die beiden Fragen Haben sie von dieser Meldung schon einmal gehört? und Glauben Sie, die Meldung entspricht der Wahrheit? wurden jeweils mittels der Ausprägungen »Nein«, »Nicht Sicher« und »Ja« operationalisiert. Auf dieser Grundlage wurde für alle 12.252 Fälle eine Rangkorrelation zur Überprüfung des Zusammenhangs zwischen der Bekanntheit einer Nachricht und dem Glauben, ob diese Nachricht der Wahrheit entspricht, durchgeführt. Zunächst einmal unabhängig davon, ob die Meldung eine Falschmeldung oder eine wahre Meldung ist, korrelieren die Bekanntheit und die 
Glaubwürdigkeit einer Nachricht höchstsignifikant, $(r s=.397, p=.000, N$ $=12.252$ ). Die positive Korrelation bringt dabei zum Ausdruck, dass je eher eine Meldung bekannt ist, desto eher ihr geglaubt wird (mittlere Effektstärke).

\section{Tabelle 1: Bekanntheit und Glaubwürdigkeit einer Meldung}

\begin{tabular}{|c|c|c|c|c|}
\hline $\begin{array}{l}\text { Bekanntheit / } \\
\text { Glaubwürdigkeit }\end{array}$ & $r_{s}$ & $p$ & $N$ & Effektstärke \\
\hline Insgesamt & .397 & .000 & 12.252 & Mittlerer Effekt \\
\hline Fake News & .218 & .000 & 6.126 & Schwacher Effekt \\
\hline Wahre Meldungen & .562 & .000 & 6.126 & Starker Effekt \\
\hline
\end{tabular}

Betrachtet man zusätzlich die Effektstärke für wahre und falsche Meldungen, so sieht man, dass dieser Zusammenhang nicht vollständig unabhängig von der Richtigkeit ist. Die Studie von Pennycook et. al. (2017), die dieser Hypothese zu Grunde liegt, hat diesen Zusammenhang bereits angedeutet. Aber selbst für erwiesene Falschmeldungen ist dieser Effekt messbar. Deshalb kann $\mathrm{H} 1$ angenommen werden, und zwar nicht nur dergestalt, dass die Vertrautheit einer Nachricht beeinflusst, ob diese geglaubt wird, sondern auch, dass dies unabhängig vom Wahrheitsgehalt gilt. Hier wird der Mere Exposure-Effekt wirksam, der besagt, dass die frühere Konfrontation mit einem Reiz schon die hinreichende Bedingung dafür ist, dass dieser bei einer späteren Wiederholung positiv bewertet wird.

\section{H2: Es besteht ein positiver Zusammenhang zwischen höherer Bildung und dem Erkennen von »Fake News«.}

Mithilfe der dichotom ausgeprägten Variable »gut/schlecht im Erkennen von Fake News« sollte durch einen Mann-Whitney-U-Test festgestellt werden, ob die zentralen Tendenzen der beiden unabhängigen Stichproben verschieden sind. Bei Begutachtung der mittleren Ränge wird deutlich, dass die beiden untersuchten Gruppen eine unterschiedliche zentrale Tendenz aufweisen, also ein signifikanter Mittelwertunterschied vorliegt. Auch bei weiterer Betrachtung der asymptotischen Signifikanz $(z=-5.134, p=.000)$ lässt sich darüber hinaus folgern, dass sich die beiden untersuchten zentralen Tendenzen unterscheiden (Mann-Whitney-U-Test: $U=102770.000$, $\mathrm{p}=.000)$.

Um dies ferner statistisch zu fundieren und die genaue Effektstärke nachzuweisen, wurde eine Rangkorrelationsanalyse nach Spearman (parameter- 
frei) durchgeführt. Danach korreliert die Bildung mit dem Erkennen von »Fake News« positiv in signifikanter Weise, wenn auch schwach: Spearman-Rho $(1.006)=.162, p<.01$.

Obwohl die Stichprobe zu rund 80 Prozent aus Teilnehmern bestand, die mindestens die Fachhochschul- oder Hochschulreife erreicht hatten und Personen mit geringerer formaler Bildung entsprechend unterrepräsentiert sind, kann die Hypothese vorläufig angenommen werden.

H3: Je höher das Alter der Befragten ist, desto eher sind sie in der Lage, "Fake News" zu identifizieren.

Zur Prüfung des Zusammenhangs zwischen Alter und der Fähigkeit, »Fake News« zu erkennen, wurde erneut mittels des oben beschriebenen dichotomisierten Scores und der Variable Alter ein Mann-Whitney-U-Test durchgeführt, der wiederum eine asymptotische Signifikanz offenbart, welche mittels einer Korrelation nach Spearman ebenfalls einen schwach positiven Zusammenhang dokumentiert (Spearman-Rho (1.021) $=.100, p<.01)$.

Aber auch ohne die Dichotomisierung der Score-Variable für das Erkennen von »Fake News« - also unter Verwendung der absoluten Zahl der erkannten Meldungen - konnte ein signifikanter schwacher positiver Zusammenhang nachgewiesen werden (Pearson $r=.081, p<.01$ ). Daraus folgt, dass in der vorliegenden Stichprobe ältere Befragungsteilnehmer »Fake News « besser identifizieren und einordnen konnten als jüngere und H3 vorläufig angenommen ist.

2020 wurde nicht mit der Score-Variablen für das Erkennen von »Fake News«, sondern mit einer Score-Variablen für das Erkennen von »Fake News « und wahren Nachrichten gearbeitet; dadurch stieg auch der Anteil der Teilnehmer, die »schlecht im Erkennen« sind, von 55 Prozent (2017) auf nun 65 Prozent. Auf der Basis einer logistischen Regressionsanalyse (Regressionskoeffizient $B(1.006)=.003, p=.56)$ konnte kein Zusammenhang zwischen Alter und Identifizierung von »Fake News« festgestellt werden.

H4.1: Je höher der Medienkonsum der Befragten, desto eher sind sie in der Lage, »Fake News« zu identifizieren.

Zum Test dieser Hypothese wurde die skaliert abgefragte Nutzungsintensität für die Mediengattungen Zeitung, Radio, Fernsehen und das Internet zu einer weiteren Score-Variablen umcodiert. Die daraus entstandene Variable Mediennutzung wurde ebenfalls mit der dichotomisierten Score-Variablen 
zum Erkennen von »Fake News« in Beziehung gesetzt und statistisch getestet. Alle Tests zeigen ganz schwache Zusammenhänge an (Pearson $r=$ $.059, p=.06$; Spearman-Rho $=.051, p=.101)$, sind aber nicht signifikant. Selbst der schlichtere normierte Kontingenzkoeffizient von .338 ist statistisch nicht signifikant $(p=.059)$. Eine detaillierte Analyse zeigt aber, dass eine überdurchschnittliche Nutzung des Internets eine höhere Mediennachrichtenkompetenz auszubilden scheint: Es zeigt sich hier ein schwacher $\mathrm{Zu}$ sammenhang nach Pearson $(r=.102, p=.001)$. Dennoch muss auf Basis der vorliegenden Daten die in H4.1 formulierte Annahme: »Je höher der Medienkonsum der Befragten ist, desto eher sind sie in der Lage, »FakeNews« zu identifizieren«, abgelehnt werden. In der Studie 2020 wurde deshalb untersucht, ob speziell die Online-Nutzung einen Effekt auf die Fähigkeit hat, »Fake News« zu identifizieren. Es wurden auf der Basis der skalierten Nutzung zwei Gruppen gebildet: Eine die das Internet unterdurchschnittlich und eine, die das Internet überdurchschnittlich nutzt. Der $\mathrm{Chi}^{2}{ }^{2}$ Test konnte keinen Effekt zwischen hoher Online-Nutzung und Erkennen von »Fake News« zeigen $\left(\chi^{2}(16, \mathrm{~N}=663=11.20, p=.79)\right.$, während die schwache Onlinenutzung erstaunlicherweise einen schwachen bis mittleren Zusammenhang andeutet $\left(\chi^{2}(60, \mathrm{~N}=361)=100.91, p=.000\right)$.

H4.2: Die Art der genutzten Online-Medien zur politischen Information hat einen Einfluss auf die Fähigkeit, »Fake News" zu identifizieren.

Um die einzelnen Einflüsse verschiedener Online-Quellen der politischen Information statistisch zu untersuchen, wurden die einzelnen Variablen mit der Variablen zum Erkennen von »Fake News« im $\mathrm{Chi}^{2}$ gekreuzt. Für die Beantwortung der Hypothese lässt sich zusammenfassend sagen, dass die Nutzung von Online-Angeboten von Rundfunkanbietern (zum Beispiel Tagesschau oder BR24) und dem Mikroblogging-Dienst Twitter einen Zusammenhang mit dem Erkennen von »Fake News« hat, der statistisch signifikant ist. Nicht die Höhe des Medienkonsums, sondern die gezielte Nutzung von Onlinequellen zum Zweck der politischen Information korreliert also mit der Fähigkeit, »Fake News« zu identifizieren. 
Tabelle 2: Einfluss von Onlinequellen zur politischen Information

\begin{tabular}{lccc} 
Online-Quelle & $\chi^{2}$ & Cramer $V$ & $p$ \\
\hline Online-Angebote (Print) & 8,25 & .090 & .509 \\
Online-Angebote (RF) & $\mathbf{1 9 , 8 3}$ & $\mathbf{. 1 3 9}$ & $\mathbf{. 0 0 1 9}$ \\
Soziale Netzwerke & 9,63 & .097 & .381 \\
Messaging-Dienste & 2,91 & .053 & .968 \\
Twitter & $\mathbf{2 3 , 4 1}$ &. $\mathbf{1 5 1}$ & $\mathbf{. 0 0 5}$ \\
News-Aggregatoren & 14,57 & .119 & .103 \\
Online only & 4,40 & .066 & .883 \\
Videoportale & 12,27 & .109 & .198
\end{tabular}

H5.1: Es besteht ein positiver Zusammenhang zwischen politischem Engagement und dem Erkennen von »Fake News«.

Das politische Engagement wurde über die vier Fragen »Sind Sie Mitglied einer politischen Partei?«, »Besuchen Sie politische Veranstaltungen?«, »Haben Sie sich bei früheren Wahlen Parteiprogramme durchgelesen?« und »Haben Sie sich für die Bundestagswahl 2017 bislang Parteiprogramme durchgelesen?« abgefragt. Aus den dichotomen Antwortoptionen wurde eine Score-Variable gebildet, die mit einer Faktorenanalyse (Rotationsprinzip Varimax, Extraktionsprinzip Hauptkomponentenanalyse) positiv auf die Ladung aller Variablen auf einer Komponente geprüft und so statistisch abgesichert wurde. Die einzelnen Variablen wurden im Anschluss mit drei unterschiedlichen Faktoren gewichtet und die finale Score-Variable für das politische Engagement mit der Score-Variablen für das Erkennen von »Fake News« korreliert. Es zeigt sich ein schwacher positiver Zusammenhang zwischen dem politischen Engagement und dem Erkennen von »Fake News « (Spearman-Rho(1021) = .186, $p<.01)$, der sich auch in einer ähnlichen Größenordnung ergibt, wenn man die beiden Variablen Dummy-codiert (dichotomisiert), um den Einfluss möglicher Unterschiede zwischen den einzelnen Stufen einer Variable auszuschließen (Spearman-Rho(1021) $=.142, p<.01)$. Hypothese 5.1 wird folglich angenommen.

H5.2: Die politische Gesinnung beziehungsweise Orientierung hat Einfluss auf das Erkennen von »Fake News«.

Um festzustellen, ob die politische Gesinnung einen Einfluss auf das Erkennen von »Fake News« besitzt, wurden die Befragten aufgrund ihrer 
Angaben zur Parteinähe auf ein Links-Rechts-Spektrum der politischen Orientierung eingestuft. Nur 809 der 1.021 Teilnehmenden machten hier Angaben. Der Durchschnitt der politischen Gesinnung liegt ganz leicht links der Mitte (Median $=3, \mathrm{IQR}=2-5$, range $=1-6, \mathrm{n}=809)$. Beim Betrachten der Korrelation der politischen Gesinnung und der Fähigkeit zum Erkennen von »Fake News« lässt sich ein schwacher Zusammenhang erkennen (Spearman-Rho(809) $=-.180, p<.01)$. Dieser Interaktionseffekt wird noch deutlicher bei der Korrelation der politischen Gesinnung und der genauen Anzahl der erkannten »Fake News« (Spearman-Rho(809) = -.218, $p<.01)$. Hypothese 5.2 kann auf Basis der statistischen Prüfung ebenfalls angenommen werden.

H6.1: Die Befragten sind der Ansicht, dass sie selbst weniger von »Fake News" beeinflusst werden als die restliche Bevölkerung.

und

H6.2: Die Befragten sind der Ansicht, dass ihr direktes soziales Umfeld weniger von »Fake News« beeinflusst wird als die restliche Bevölkerung.

Mit Blick auf die Bundestagswahl 2017 wurde zunächst analysiert, ob die Befragungsteilnehmer der Meinung sind, dass sie fallweise schon einmal von Falschmeldungen beeinflusst wurden. 13 Prozent der Befragten bejahten dies, 55 Prozent verneinten die Frage, gut ein Drittel konnte dies nicht sicher sagen. Auch die Frage, ob es eventuell häufiger vorgekommen sei, dass man sich eine Meinung zu einem Thema gebildet habe und sich später aufgrund einer gewissen Nachricht herausstellt, dass alles anders sei, bejahten gut zehn Prozent der Teilnehmer, während sich knapp zwei Drittel des Samples als resistent bezeichneten.

Zur Testung des Second-Person- und Third-Person-Effekts wurde aber konkret der vermutete Einfluss von »Fake News « auf diese spezielle Bundestagswahl erfragt. Schon an den Häufigkeitsverteilungen (Tabelle 3) ist zu erkennen, dass die Befragten $(\mathrm{n}=$ 976/984) den Einfluss von $»$ Fake News« auf sich selbst als geringer einschätzen als auf das persönliche Umfeld und erst recht auf die Gesamtbevölkerung. 
Tabelle 3: Einfluss von »Fake News« auf Bundestagswahl 2017 - TPE

\begin{tabular}{lccc}
$\begin{array}{l}\text { »Fake News« können Wahl- } \\
\text { verhalten beeinflussen... }\end{array}$ & ja & nein & teils, teils \\
\hline bei mir selbst & 13,4 & 75,2 & 11,4 \\
in meinem persönlichen Um- & 31,5 & 42,6 & 25,9 \\
feld & 67,9 & 9,1 & 23 \\
in der Gesamtbevölkerung & & \\
\hline
\end{tabular}

Zur statistischen Absicherung dieses Befundes wurden bivariate ProduktMoment-Korrelationen zwischen den entsprechenden Variablen berechnet. Für die Variablen Einfluss von »Fake News « (FN) auf eigene Wahlentscheidung und Einfluss von FN auf Wahlentscheidung der Gesamtbevölkerung zeigt sich ein mittelstarker Zusammenhang $(r=.32, p<.001)$. Für die Variablen Einfluss von FN auf Wahlentscheidung des Umfelds und Einfluss von FN auf Wahlentscheidung der Restbevölkerung ergibt sich sogar ein mittelstarker bis starker Zusammenhang von $r=.50, p<.001$. Wie den beiden $\mathrm{p}$-Werten zu entnehmen ist, weisen beide Korrelationen ein sehr hohes Signifikanzniveau auf.

Tabelle 4: Pearson Korrelationstabelle zum SPE und TPE

\begin{tabular}{lcc}
\hline & $\begin{array}{c}\text { Einfluss von FN auf ei- } \\
\text { gene Wahlentscheidung }\end{array}$ & $\begin{array}{c}\text { Einfluss von FN auf } \\
\text { Wahlentscheidung d. } \\
\text { Umfelds }\end{array}$ \\
\hline $\begin{array}{l}\text { Einfluss von FN auf } \\
\text { Wahlentscheidung der } \\
\text { Restbevölkerung }\end{array}$ & $.321^{* *}$ & $.503 * *$ \\
\hline$* * p<.001$ & & \\
\hline
\end{tabular}

Die ermittelten Zusammenhänge sowie die Analyse der Kreuztabellen lassen darauf schließen, dass tatsächlich ein starker Second-Person-Effekt und Third-Person-Effekt hinsichtlich der Beeinflussung durch »Fake News« wirksam wird. Diese Befunde befinden sich in großer Übereinstimmung mit den bisherigen Forschungen zu diesem Phänomen (Rössler, 2009, Dohle, 2013; Dohle, 2016; YouGov, 2017). Die Hypothesen 6.1 und 6.2 werden angenommen. 


\section{Diskussion}

Mit Hilfe einer Onlinebefragung unter 1.021 (1.042) Internetnutzerinnen und -nutzern und einem quasi-experimentellen Untersuchungsansatz wurde analysiert, welche Bedeutung »Fake News« in der wahlberechtigten Bevölkerung in Deutschland zugeschrieben wird (Problemsensibilität), inwieweit die Fähigkeit bei Internetnutzern ausgebildet ist, Falschmeldungen von echten Nachrichten unterscheiden zu können (Problemlösungskompetenz) und welche Faktoren das Erkennen von »Fake News« begünstigen (Problembewältigung). Folgende Limitationen sind hinsichtlich der Diskussion der Befunde zu berücksichtigen:

Die Studie ist als selbstrekrutierende Onlinebefragung mit quasi-experimentellem Design nicht bevölkerungsrepräsentativ. Sie ist mit Blick auf die Grundgesamtheit hinsichtlich der Merkmale Alter, Bildung und regionale Herkunft dergestalt verzerrt, dass formal höher Gebildete, Menschen zwischen 20 und 39 Jahren und Menschen, die in Bayern leben, überdurchschnittlich vertreten sind. Sie dürfte typisch für ein studentisches und überwiegend junges akademisches Milieu sein, das sowohl wahlberechtigt als auch wahlwillig ist. Schlüsse auf die Gesamtbevölkerung sind insofern nicht zulässig.

Obwohl der Kern des Fragebogens nahezu identisch ist, sind die beiden Feldphasen im Juli 2017 und Januar 2020 nur bedingt vergleichbar, da sowohl beim Stimulusmaterial als auch bei einigen wenigen Skalen geringfügige Korrekturen vorgenommen worden sind. Direkte Vergleiche haben deshalb die Ergebnisdarstellung nicht geleitet, denn das variierte StimulusMaterial und die abweichenden Formen der Datenstrukturierung im Sample 2020 sind vermutlich eher für kleinere Abweichungen verantwortlich als die beiden Erhebungszeitpunkte im Vorfeld einer Bundestagswahl (2017) und ohne Wahlkampfphase (2020).

Ungeachtet dieser methodischen Einschränkungen konnten einige stabile Befunde zur Wahrnehmung und Bewertung von »Fake News« im Rahmen dieser Studie generiert werden. Zentral ist der Zusammenhang zwischen dem Kennen von Meldungen und dem (vermeintlichen) Erkennen wahrer Nachrichten: Menschen, die mit einer Meldung schon einmal konfrontiert waren, schenken dieser mit einer höheren Wahrscheinlichkeit Glauben. Dieser Wahrheitseffekt - Illusory Truth Effect oder Mere Exposure Effectist auch dann wirksam, wenn die Meldung offenkundig unplausibel und falsch ist. In der vorliegenden Studie konnte dieser Zusammenhang zwischen Vertrautheit und Glaubwürdigkeit nicht nur für einzelne Meldungen nachgewiesen werden, sondern mithilfe statistischer Prüfverfahren für das 
gesamte Untersuchungssample. Zum Teil wird dieser Umstand dafür verantwortlich gemacht, dass es nicht ausreicht, mit Korrekturinitiativen und dem sogenannten Debunking (Entzaubern) dem Problem »Fake News« zu Leibe zu rücken, auch weil das Kenntlichmachen von »Fake News« eine geringere Verbreitung erfährt als die Falschmeldung selbst. Was erst einmal in der Welt ist, bleibt offenbar relativ robust dort.

Was begünstigt die Fähigkeit, »Fake News« als solche identifizieren zu können? Studienteilnehmer mit formal höherer Bildung waren eher in der Lage, »Fake News« zu erkennen als formal geringer Gebildete. Auch mit dem Alter steigt offenbar zumindest ansatzweise die Fähigkeit, echte Nachrichten von falschen unterscheiden zu können an. Dagegen ist eine höhere allgemeine Mediennutzung dem besseren Erkennen von Falschmeldungen nicht zuträglich, vielmehr sind Nutzer mit spezifischer Zuwendung zu Onlinemedien statistisch betrachtet besser in der Lage, »Fake News« zu identifizieren, zumindest, wenn diese Onlinemedien zur politischen Information genutzt werden. Dass hier die Nutzung von Onlinemedien aus dem Rundfunkbereich auf das Konto der Identifizierung von Falschmeldungen einzahlt, ist vermutlich der Nutzung der Nachrichtenapps von Tagesschau und BR24 geschuldet, die sich einer öffentlich-rechtlichen Sozialisation der Studienteilnehmer verdanken dürfte. Beim positiven Einfluss der Nutzung von Microblogging-Diensten dürfte eine Rolle spielen, dass Twitter zum einen von einer gewissen Informationselite genutzt wird, zum anderen verfügen Twitter-Nutzer über ein spezielles Themenwissen, da »Fake News« dort nicht nur stark verbreitet, sondern auch stark diskutiert werden.

Auch das politische Engagement und die politische Orientierung tragen dazu bei, den Wahrheitsgehalt von Nachrichten besser einschätzen zu können. Wer sich politisch informiert, engagiert, Wahlveranstaltungen besucht oder Mitglied in einer Partei ist, kann besser beurteilen, ob eine Nachricht wahr oder ein Fake ist. Studienteilnehmer mit einer eher linken politischen Orientierung waren etwas besser in der Lage, Desinformation als solche zu erkennen als Teilnehmer mit einer Affinität zum bürgerlich-konservativen Lager. Dieser Befund deckt sich - wie die meisten anderen - mit Vorläuferuntersuchungen und könnte darauf zurückzuführen sein, dass weltanschaulich progressive beziehungsweise linke Bürgerinnen und Bürger kritischer und skeptischer in ihrer Grundeinstellung sind als diejenigen mit konservativer Weltanschauung. Dass das politische Interesse und Engagement sich positiv auf die Identifizierungskompetenzen auswirken, ist für die Initiativen zur politischen Bildung eine gute Nachricht.

Insgesamt betrachtet korrelieren die soziodemographischen Variablen Alter und Bildung deutlich schwächer mit der »Fake News«-Erkennung als 
die verhaltensbezogenen Variablen »Mediennutzung « und »politisches Engagement«. Vermutlich sind hier Interaktionseffekte zwischen den Dimensionen wirksam, die jedoch nicht in einem Regressionsmodell kontrolliert werden konnten.

Prinzipiell fühlen sich die Befragungsteilnehmenden nur geringfügig von einzelnen Falschmeldungen beeinflussbar. Gleichwohl konnte in diesem Zusammenhang das Vorhandensein sowohl des Second-Person- als auch des Third-Person-Effekts nachgewiesen werden: Obwohl die wenigstens Personen sich selbst als beeinflussbar bezeichnen, glaubt ein Drittel, dass die Wahlabsichten des persönlichen Umfelds, mehr als zwei Drittel sogar, dass die Wahlabsichten der Gesamtbevölkerung von »Fake News« beeinflusst werden. Die konkreten Leistungsbefunde - nämlich eine gewisse Anfälligkeit für Desinformation (die Quote der richtig erkannten Falschmeldungen lag in beiden Feldphasen im Bereich des Zufälligen) - sprechen dafür, dass die Befürchtungen der Teilnehmer nicht übertrieben sind, dass »Fake News« die Bevölkerung der Bundesrepublik Deutschland im Zuge von Wahlen negativ beeinflussen können.

\section{Literatur}

Allcott, Hunt \& Gentzkow, Matthew (2017). Social Media and Fake News in the 2016 Election. Journal of Economic Perspective, 31(2), 211-236. DOI: 10.1257/jep.31.2.211.

Beermann, Matthias (2017). Der Krieg und die Wahrheit. Propaganda, Manipulation und Desinformation. RP Online, 1. Juli 2017. Abgerufen von http://www.rponline.de/politik/krieg-und-wahrheit-propaganda-manipulation-und-desinformation-aid-1.6918354. Abgerufen am 25. Juni 2020.

Bussemer, Thymian (2008). Propaganda. Konzepte und Theorien. Wiesbaden: VS Verlag für Sozialwissenschaften.

Chen, Yimin; Rubin, Victoria L. \& Conroy, Niall J. (2015). Deception Detection for News. Three Types of Fakes. ASIST, Proceedings of the Association for Information Science and Technology, 52(1), 1-4. DOI: 10.1002/pra2.2015. 145052010083.

Dohle, Marco (2013). Third-Person-Effekt. Baden-Baden: Nomos Verlag.

Dohle, Marco (2016). The Third-Person Effect in Communication. In: Potthoff, M. (Hrsg.), Schlüsselwerke der Medienwirkungsforschung (195-204). Wiesbaden: Springer Fachmedien Wiesbaden.

Gelfert, Axel (2018). Fake News: A Definition. Informal Logic, 38(1), 84-117. DOI: 10.22329/il.v38i1.5068.

Graeupner, Damaris \& Coman, Alin (2017). The Dark Side of Meaning-Making. How Social Exclusion Leads to Superstitious Thinking. Journal of Experimental Social Psychology, 69, 218-222, DOI: 10.1016/j.jesp.2016.10.003. 
Guess, Andrew.; Nagler, Jonathan \& Tucker, Joshua (2019). Less Than You Think. Prevalence and Predictors of Fake News Dissemination on Facebook. Science Advances, 59(1), 1-8. DOI: 10.1126/sciadv.aau4586.

Heickerö, Roland (2013). The Dark Sides of the Internet. On Cyber Threats and Information Warfare. Frankfurt am Main: Peter Lang $\mathrm{GmbH}$, Internationaler Verlag der Wissenschaften.

Keil, Lars-Broder \& Kellerhoff, Sven Felix (2006). Gerüchte machen Geschichte. Folgenreiche Falschmeldungen im 20. Jahrhundert. Berlin: Christoph Links Verlag LinksDruck GmbH.

Lee, Tien-Tsung (2010). Why They Don't Trust the Media. An Examination of Factors Predicting Trust. American Behavioral Scientist, 54(1), 8-21. DOI: 10.1177/ 0002764210376308.

Metzger, Miriam J.; Hartsell, Ethan H. \& Flanagin, Andrew J. (2015). Cognitive Dissonance or Credibility? A Comparison of Two Theoretical Explanations for Selective Exposure to Partisan News. Communication Research, 47(1), 3-28. DOI: 10.1177/0093650215613136.

Pennycook, Gordon; Cannon, Tyrone D. \& Rand, David G. (2017). Prior Exposure Increases Perceived Accuracy of Fake News. Journal of Experimental Psychology: General, 147(12), 1865-1880. DOI: 10.1037/xge0000465.

Rössler, Patrick (2009). Wie Menschen die Wirkungen politischer Medienberichterstattung wahrnehmen - und welche Konsequenzen daraus resultieren. Zum Zusammenhang von politischer Willensbildung, Second- und Third-Person-Effekten. In: Marcinkowski, F. \& B. Pfetsch (Hrsg.), Politik in der Mediendemokratie (468-495). Wiesbaden: VS Verlag für Sozialwissenschaften.

Smith, Aaron (2013). Civic Engagement in the Digital Age. Pew Research Center. Abgerufen von https://www.pewresearch.org/internet/2013/04/25/civic-engagement-inthe-digital-age/ . Abgerufen am 01. Juli 2020.

Schwartau, Winn (1996). Information Warfare. Thunder's Mouth Press, New York.

Tandoc Jr., Edson C.; Lim, Zheng Wei \& Ling, Richard (2018). Defining »Fake News«. A Typology of Scholarly Definitions. Digital Journalism, 6(2), 137-153. DOI: 10.1080/21670811.2017.1360143.

Tsfati, Yariv \& Ariely, Gal (2013). Individual and Contextual Correlates of Trust in Media Across 44 Countries. Communication Research, 41(6), 760-782. DOI: 10.1177/0093650213485972.

Tsfati, Yariv \& Cappella, Joseph N. (2003). Do People Watch what they Do Not Trust? Exploring the Association between News Media Skepticism and Exposure. Communication Research, 30(5), 504-529. DOI: 10.1177/0093650203253371.

YouGov (2017). »Alles Fake?!«. Fake News aus Sicht deutscher Wähler. Abgerufen von https://campaign.yougov.com/DE_2017_08_Political_Fake_News.html. Abgerufen am 29. Juni 2020.

Van Herpen, Marcel H. (2016). Propaganda und Desinformation. Ein Element »hybrider« Kriegführung am Beispiel Russland. Aus Politik und Zeitgeschichte, 3536/2016, 16-21. 
Wardle, Claire (2017). Fake News - Es ist kompliziert. First Draft, 17. März 2017. Abgerufen von https://de.firstdraftnews.org/fake-news-es-ist-kompliziert/. Abgerufen am 29. Juni 2020.

Zimmermann, Fabian \& Kohring, Matthias (2018). »Fake News« als aktuelle Desinformation. Systematische Bestimmung eines heterogenen Begriffs. Medien \& Kommunikationswissenschaft, 66(4), 526-541. DOI: 10.5771/1615-634X-2018-4-526. 
